\title{
Kognitive Störungen zwischen Psychiatrie und Neurologie
}

Störungen des Denkens und des Verhaltens treten im Rahmen einer Vielzahl von neurologischen und psychiatrischen Erkrankungen zutage. Sie können entweder Folge einer morphologisch faßbaren Hirnschädigung sein oder aus einer Imbalance der an den komplexen Hirnfunktionen beteiligten Neurotransmitter resultieren. Bei letzteren muss neben der genetischen Prädisposition auch die Umwelt im Sinne eines biopsychosozialen Modells berücksichtigt werden.

Verhaltensneurologie und klinische Neuropsychologie haben in den letzten Jahren zunehmend Eingang in neurologische, neurochirurgische und psychiatrische Diagnostik-, Behandlungs- und Forschungskonzepte gefunden. Ihre praktischen Schwerpunkte liegen in der Erfassung und Behandlung von Störungen im Bereich des Denkens und Handelns, der Sprache, der Aufmerksamkeits- und Gedächtnisprozesse sowie der Wahrnehmung, aber auch geistig-seelischer und psychosozialer Veränderungen. Zur Erfassung und Evaluation der genannten Störungen bedienen sie sich dabei eines umfangreichen Untersuchungs- und Methodenrepertoires. Entsprechend der vielschichtigen Symptomatik hirnverletzter und -erkrankter Patienten gestaltet sich der Arbeitsbereich im klinischen Sektor interdisziplinär, im engen Austausch zwischen den Fachgebieten der Neurologie und Psychiatrie, der Neurochirurgie, aber auch der inneren Medizin. Schließlich hat die verhaltensneurologisch-neuropsychologische Diagnostik, auch unter qualitätssichernden Aspekten einen zunehmenden Stellenwert in der interventionellen Neuroradiologie im Rahmen des neuropsychologischen Monitorings, z. B. bei selektiven Gefäßokklusionen oder bei selektiven Natrium-Amytal-Tests (Wada-Test). Insbesondere durch die Entwicklung moderner dynamischer bildgebender Verfahren wie der funktionellen Magnetresonanztomografie (fMRT) eröffnen sich durch die Kombination dieser apparativen Verfahren mit kognitiven Stimulationsparadig- men sowohl Möglichkeiten der zusatzdiagnostischen Optimierung bereits etablierter Verfahren, die dem Patienten direkt zugute kommen (z.B. „schonendes Operieren“ durch die exakte Beschreibung und Markierung sprachrelevanter Hirnregionen) als auch Chancen zur Bearbeitung von interdisziplinären Fragestellungen mit grundlagenwissenschaftlicher Relevanz.

Unabhängig davon, ob diese Spezialdisziplin von Seiten der Ärzte (Verhaltensneurologie) oder von Seiten spezialisierter Psychologen (Neuropsychologie) vertreten wird, bedient sie sich eines interdisziplinären Methodenrepertoires, um Zusammenhänge zwischen Gehirnfunktionen und Verhalten herzustellen. Typische Forschungsschwerpunkte beziehen sich auf Aufmerksamkeit, Gedächtnis, Sprachverarbeitung, visuelle und akustische Verarbeitung, integrative Steuerung motorischer Prozesse und soziale Kognition.

Insbesondere durch die Entwicklung moderner struktureller und dynamischer bildgebender Verfahren gelingt es zunehmend, das Gehirn im experimentellen Sinne sowohl als „abhängige“ als auch als „unabhängige“ Variable zu betrachten. Phänomene wie „Bewusstsein“, die bislang nur philosophisch betrachtet wurden, werden durch die Kombination interdisziplinärer Methoden - zumindest in ihren Teilkomponenten - einer experimentellen Überprüfung zugänglich. Die Beschreibung und Untersuchung struktureller Aspekte und funktioneller Zusammenhänge von gedächtnis- und sprachrelevanten Hirnstrukturen sowohl bei hirngesunden als auch hirngeschädigten Probanden mittels neuropsychologischer Methoden einerseits und der modernen Bildgebung andererseits sind ein Beispiel für die systematische, wissenschaftliche Annäherung zur Erklärung komplexen menschlichen Verhaltens. In diesem Schwerpunktheft werden verschiedene Aspekte dieser Teildisziplin synoptisch dargestellt und anhand klinisch relevanter Patientengruppen verdeutlicht.

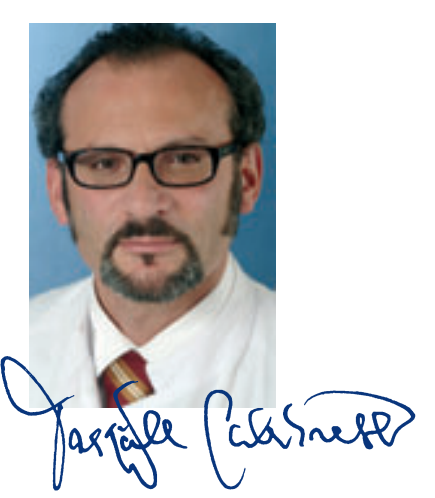

Prof. Dr. rer. nat. Pasquale Calabrese, Bochum 